\title{
Management of Mandibular Fractures from Penetrating Trauma
}

\author{
Elizabeth Hoddeson ${ }^{1}$, Eric Berg ${ }^{2}$ and Charles Moore, ${ }^{*}$ \\ ${ }^{1}$ Department of Otolaryngology, Emory University Hospital, 1365 Clifton Road, Atlanta, GA 30322, USA \\ ${ }^{2}$ Department of Otolaryngology, Children's Hospital of Philadelphia, $34^{\text {th }}$ Street \& Civic Center Blvd., Philadelphia, PA \\ 19104, USA
}

\begin{abstract}
Gunshot wound injuries to the face result in a varied scope of traumatic injuries. Mandible fractures may occur through this mechanism of injury, however when caused by firearms, the patterns of fracture and overall composite defects resulting are unique. The velocity of the weapon and the distance from which the injury occurred often determines the extent of injury produced from the penetrating force. A review of the management of mandibular fractures from penetrating trauma is presented. Operative and reconstructive options including advantages and disadvantages are discussed for consideration to produce the optimal result for rehabilitation of the injured patient.
\end{abstract}

Keywords: Mandibular Fractures, Penetrating Trauma, firearms, velocity of the weapon.

\section{INTRODUCTION}

Penetrating injuries are defined as those that violate mucosal or skin barriers to enter the body, and can be caused by knives, bullets, shrapnel, or other objects. The type of injury is often determined by the velocity of the weapon and the distance from which the injury occurred [1]. The true incidence of gunshot wound injuries to the face is unknown; one retrospective study over a 4 year period showed that the face was injured in $6 \%$ of all gunshot wound victims at one trauma center [2].

Gunshot wound injuries to the face result in a varied scope of traumatic injuries including soft tissue, vascular, skeletal, orbital, cervical spine, thoracic, and intracranial. Overall, mandible fractures are very common, however when caused by firearms, the patterns of fracture and overall composite defects resulting are unique. Among mandible fractures, condylar fractures are common, between $17.5 \%$ $52 \%$ of all mandible fractures [3]. Condylar fractures are classified in two ways: by anatomic level as condylar head (intracapsular), condylar neck (extracapsular), and subcondylar, and also as undisplaced, deviated, displaced (with medial or lateral overlap or complete separation), and dislocated (outside the glenoid fossa) [3].

Condylar fracture reconstruction can be approached with a variety of materials including free bone grafts, metal prosthesis, and pediculate bone cancellous marrow grafts. The choice of reconstruction material is often dependent on the specific nature of the fracture and coincidental bone defect as well as the overall rehabilitative potential of the

*Address correspondence to this author at the Department of Otolaryngology, Emory University Hospital, 1365 Clifton Road, Ste. 2300, Atlanta, GA 30322, USA; Tel: 404-778-3381; Fax: 404-616-8202;

E-mail: cemoore@emory.edu patient for mastication, occlusion, and function. Presence of a large bone defect limits traditional reconstructive options. Here we present a unique approach to the repair of such a defect.

\section{CASE PRESENTATION}

A 27 year old otherwise healthy male presented to Grady Memorial Hospital in May, 2009 subsequent to suffering a gunshot wound to the left face one inch anterior to the tragus. The list of injuries incurred included vertebral artery thrombus, external carotid artery thrombus, left $\mathrm{Cl}$ spine fracture, left facial nerve paralysis, left external auditory canal lacerations, and multiple facial fractures including subluxed left condylar fracture and completely disrupted and fragmented left ramus fracture. He was intubated in the operating room emergently, a heparin drip was initiated, and local wound care with iodoform packing was initiated for the gunshot wound. Definitive surgical repair of mandible defect was deferred until the patient's clinical status was stabilized and coexisting injuries were managed, about six weeks in total. This time was utilized for operative planning. Following his initial trauma the patient was placed in a cervical collar for six weeks; on the completion of this period antero-posterior flexion extension radiographs were obtained by the orthopedic spine physicians. The patient had no evidence of instability or fractures on imaging and no clinical pain or paresthesias with bending the neck. The cervical collar was removed and the patient cleared for normal mobility prior to operative intervention on the mandible. The patient was admitted to the hospital preoperatively in order to bridge his anticoagulation, which is prescribed by the trauma surgeons indefinitely, with heparin.

Further review of the radiographic images demonstrated a comminuted defect of the left mandible extending from the mid-body through the condylar neck (Fig. 1). The native 


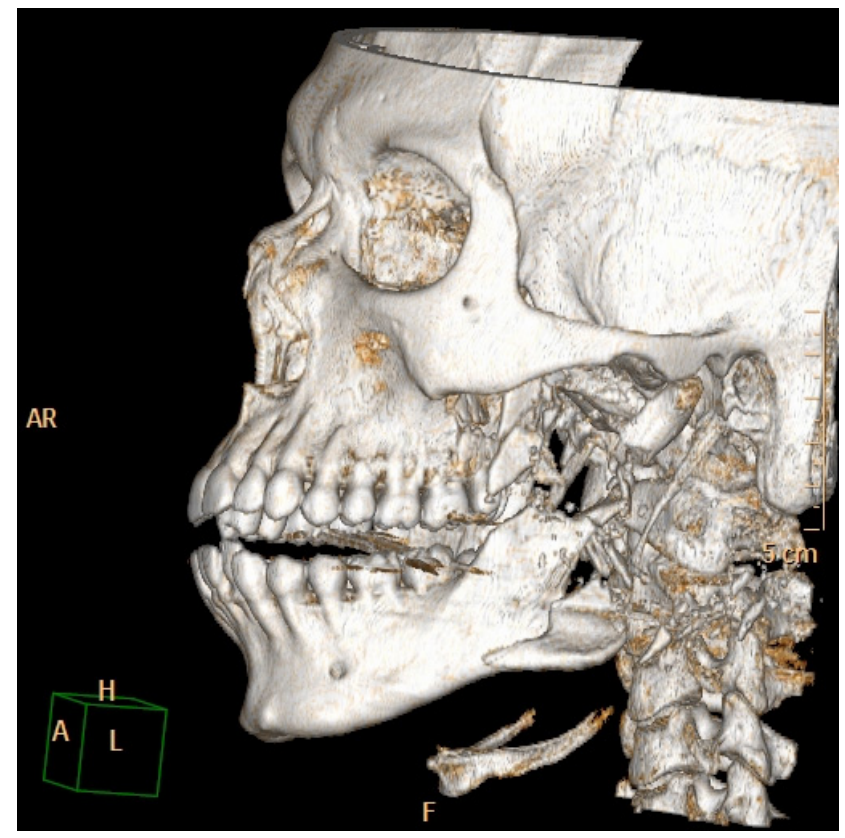

Fig. (1). Three-dimensional reconstruction of computed tomography of mandible demonstrating comminuted defect.

condyle was displaced medially and the remaining fragments were not usable due to their extensive fragmentation. A custom prosthesis was designed based on a threedimensional model of the patient's skeletal defects utilizing Medical Modelling Software (C). The prosethesis was produced by Biomet $(\mathrm{C}$, and the total cost of design and production was $\$ 9500.00$ (Figs. 2, 3). Bone support from an iliac crest bone graft was considered during the reconstruction planning stages.

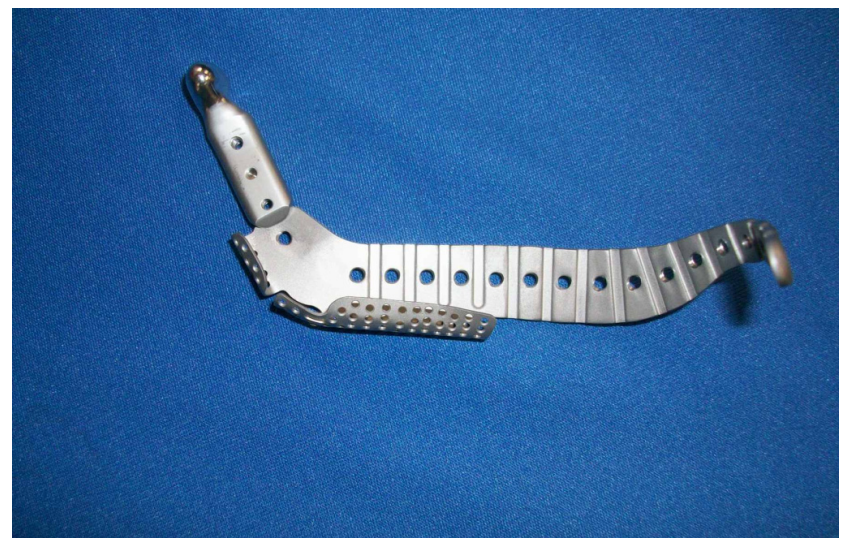

Fig. (2). Custom-designed mandibular prosthesis.

The patient was taken to the operating room where an external approach to the mandible to the level of the glenoid fossa was utilized. The patient had extensive scarring, which made identification of normal tissue planes and anatomic landmarks difficult (Fig. 4). The inferior division of the facial nerve was absent, however the superior division was identified and protected. The native condylar head was located and removed from its dislocated position medial to the glenoid fossa. Multiple small bone fragments were removed from the region of the ramus, but a moderately large piece of inferior ramus was found to have formed a bony union with the body of the mandible. Using a reciprocating saw, this fragment was shaved in order to fit within the tray of the prosthesis. This precluded the need for cadaveric or autologous bone grafting. The patient was placed into maxillary-mandibular fixation to ensure proper occlusion. At this point, the implant was positioned with the condylar prosthesis securely inside the glenoid fossa and the tray flush with native mandible body and parasymphysis anteriorly (Fig. 5). It was secured in place using multiple locking bone screws. An unsupported portion of the prosthesis at the angle of the mandible was bridged using bone matrix protein and crushed bone fragments. The wound was irrigated and closed in a standard fashion with a Jackson-Pratt drain in place.

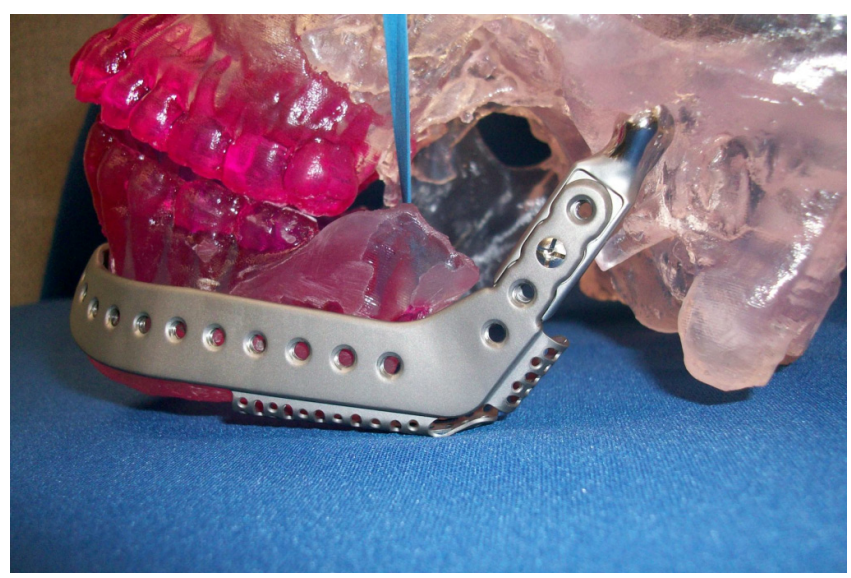

Fig. (3). Custom-designed mandibular prosthesis in position on three-dimensional model.

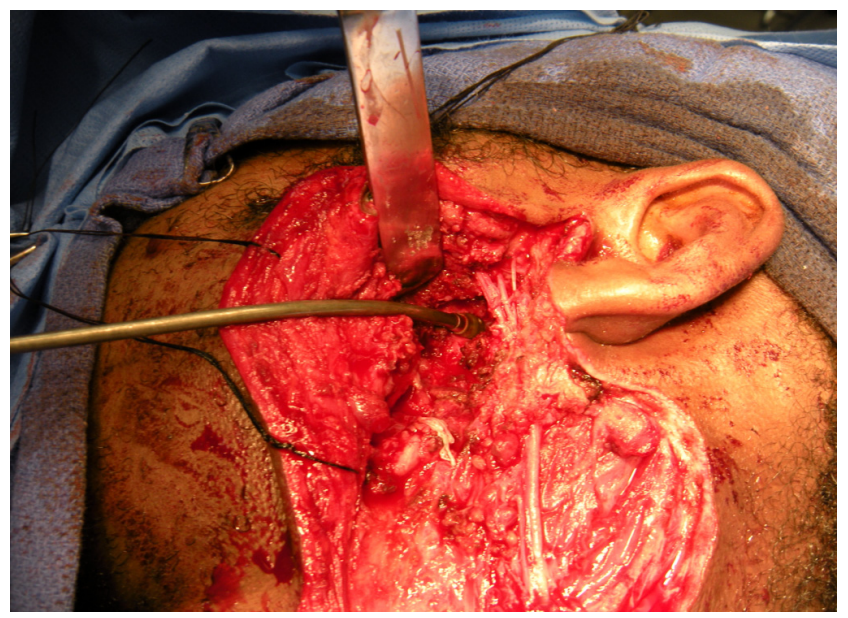

Fig. (4). Dissection bed after removal of bone fragments. Suction tip points to glenoid fossa with superior division of facial nerve lying laterally.

The patient had an uneventful hospital post-operative course and was discharged home with maxillary-mandibular fixation wires remaining intact. The wires and screws securing maxillary-mandibular fixation were removed six weeks post-operatively as the patient was in normal dental occlusion and a panorex film demonstrated that the prosthetic condyle was in proper position within the glenoid fossa. The patient had improvement in his trismus as compared to pre-operative measurements immediately after 


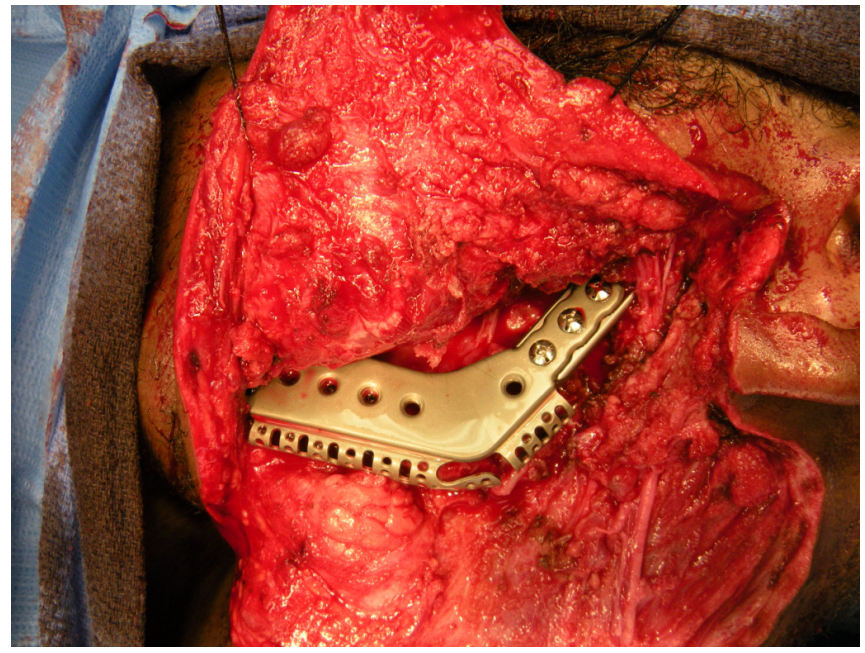

Fig. (5). Prosthesis positioned in glenoid fossa, secured to native mandible anteriorly.

the wires were removed with $1.2 \mathrm{~cm}$ opening between the central incisors. He was fitted with a Dyna splint and over the subsequent nine months improved to $4 \mathrm{~cm}$ of opening. He continued to work with a physical therapist specializing in temperomandibular joint disorders throughout the first two years following surgery. A CT face obtained three years following surgery demonstrates displacement of the condylar prosthesis anteriorly and inferiorly from the glenoid fossa, a finding which had been present since the first post-operative CT obtained at six months post-operatively, and resorption of the bone chips and graft material placed during surgery. The patient has normal eye closure but persistent paralysis of the lower division of the facial nerve following the trauma. $\mathrm{He}$ is satisfied with the cosmetic outcome of the mandibular contour, but does suffer from chronic pain in his left face, back, and shoulder following this traumatic event. His pain is managed by a pain physician.

\section{DISCUSSION}

There are multiple operative approaches to management of mandible fractures well-described in the literature. Traumatic or iatrogenic mandible defects present a more difficult challenge, often requiring a creative approach by the surgical team. This becomes substantially more complex when there is involvement of the condyle. Regardless of the nature of the defect, the ideals for mandible reconstruction remain constant: to provide an anatomical reconstruction with sufficient height and adequate native muscle attachment to allow for normal function.

Approaches described have been utilized in the past both alone and in combination. In certain circumstances patients with small mandible defects, particularly in the case of malignancy can be left to swing and have satisfactory functional outcomes. This is often desirable when radiation therapy has already been delivered or is anticipated, as it significantly impairs wound healing and increases postoperative graft failure and infection rates.

Perhaps the most simple technique involves application of a reconstruction plate. These plates depend upon bone support from cadaveric, autologous, or native sources. Locking screws are utilized to minimize compression of underlying bone and to optimize vascularity surrounding the graft as to permit stabilization without precise alignment of the plate against the bone [4]. With a small defect, less than one centimeter, a plate can be used without a bone graft. Advantages of this technique include decreased operative time and a viable alternative for patients where poor ability to perfuse and heal microvascular anastamoses is anticipated. Disadvantages include risk of wound dehiscence with plate exposure which increases in the setting of radiation therapy to $20-48 \%$, infection resulting most often from loosening or breakage of screws, plate breakage, and imaging difficulties.

Pediculate bone cancellous marrow grafts are based upon the Axhausen theory of osteogenesis in which surviving transplanted cells proliferate and form random new osteoid dependent upon the spatial orientation of the grafted tissue and the resorb and remodel into mature osteons with an organized structure [4]. Advantages of this technique include a high rate of osteogenesis, low rate of surgical complications, early resumption of function. Disadvantages resemble those of reconstruction plates with wound dehiscence, tray exposure, infection, and interference with implant rehabilitation requiring ultimate removal of the tray once mature bone has formed.

Larger defects are better served using vascularized bone flaps [4, 5]. Autologous bone sources include free bone grafts, pedicled bone grafts, and microvascular free flaps. This becomes more complicated and technically difficult when the defect involves the condyle, although rib grafts shaped to from a neo-condyle have been described. Free bone grafts are effective for defects less than 5 centimeters in width with surrounding soft tissue uncompromised. Success is highly dependent on revascularization at the recipient site and any micro-movement from non-rigid fixation of the graft jeapordizes viability. There is very low morbidity at the donor site for free bone grafts, most frequently harvested from the ipsilateral iliac crest. Pedicled bone grafts have essentially been replaced by microvascular free flaps. The major disadvantages of this approach that are overcome by the free flap include the very thin width of grafted bone which is insufficient to support prostheses, a bone longer than 5 centimeters is not always available or sufficiently vascularized to be viable, and very poor asthetic results. The major microvascular free flaps described and regularly used for mandible reconstruction include the radial, scapula, iliac crest, and fibular. The major advantages of this approach are repair of soft tissue and bone defects simultaneously from one donor site with very high success rates even in the setting of radiation therapy, and satisfactory functional outcomes. Disadvantages include prolonged operative time, varying rates of donor site morbidity, and the fact that outcome success is based largely upon hospital infrastructure able to support both the high demands intra- and postoperatively for these patients.

More recently transport disc distraction osteogenesis is described in the literature as an application for mandible reconstruction after many years of use in long bones [4, 6]. In this technique new bone formation is induced by an opening created by a distraction device between two bony surfaces. Advantages include the fact that regenerated bone has similar dimensions to preexisting mandible, including the ability for the inferior alveolar canal to reccanulate, there is no donor site, and facilitates use of dental implants. 
Disadvantages include difficulty in regenerating complicated curves in symphyseal and condylar defects, expensive, and difficult patient compliance issues.

Tissue engineered transplants are described in the literature. There are several ongoing studies surrounding this technique and several successful case reports described utilizing this technology. They are not utilized in general practice at this point in time.

Modern imaging and 3-dimensional modeling techniques to create a custom prosthesis have facilitated a new approach to large mandible defects involving multiple subsites of the mandible. An exact model of the mandible with the present defect can be manufactured based on a three dimensional reconstruction of the patient's computerized tomography scan. The surgeon can use this model to bend a reconstruction plate pre-operatively in order to decrease operating time and enhance precision of the plate contour. Alternatively, this technology can facilitate creation of a custom plate with or without an included prosthetic condyle with prearranged dimensions for creating osteotomies in grafted bone to facilitate an exact fit. Advantages to such a technique are multiple and include decreased operating time, improved asthetic result, more accurate alignment of native and grafted bone segments presumably improving vascularization and viability, and decreased surgeon fatigue intraoperatively. The major disadvantages are cost and preoperative time requirement in order to create the model and custom plate. Our case demonstrates the success of such an approach.

\section{CONFLICT OF INTEREST}

The authors confirm that this article content has no conflict of interest.

\section{ACKNOWLEDGEMENTS}

Declared none.

\section{REFERENCES}

[1] Johnson JV. Gunshot wounds to the face. J Trauma 2007; 62(6 Suppl): S87.

[2] Demetriades D, Chahiuan S, Gomez H, et al. Initial evaluation and management of gunshot wounds to the face. J Trauma 1998; 45: 39-41.

[3] Zachariades N, Mezitis M, Mourouzis C, Papadakis D, Spanou A. Fractures of the mandibular condyle: a review of 466 cases. Literature review, reflections on treatment and proposals. J Craniomaxillofac Surg 2006; 34(7): 421-32.

[4] Kellman, Robert M. Maxillofacial trauma. In: Cummings CW, Ed. Otolaryngology Head and Neck Surgery. $4^{\text {th }}$ ed. Philadelphia: Elsevier Mosby 2005; vol. 1: pp. 602-38.

[5] Chopra S, Enepekides DJ. The role of distraction ostiogenesis in mandibular reconstruction. Curr Opin Otolaryngol Head Neck Surg 2007; 15: 197-201

[6] Yilmaz M, Vayvada H, Menderes A, Demirdover C, Kizilkaya A. A comparison of vascularized fibular flap and iliac crest flap for mandibular reconstruction. J Craniofac Surg 2008; 19(1): 227-34. 